\title{
Les enjeux critiques du roman policier
}

\author{
Ahmed Fathy Rezk* \\ drahmedrezkr@gmail.com
}

\section{Résumé}

Le roman policier offre un meilleur exemple de la relation lecture/ critique dans la mesure où il accorde une place prépondérante au lecteur chargé de recomposer l'histoire et de résoudre l'énigme.

Le roman policier représente également le mariage entre roman et critique dans le sens où il accorde une grande dimension à l'intervention des sciences humaines dans l'interprétation de l'œuvre. Nous parlons évidemment de la psychanalyse comme clé de la compréhension du roman policier et du déchiffrement de son énigme.

Loin d'être une relation bien établie, la nouvelle Le motif dans le tapis ainsi que La lettre volée, et un drame bien parisien, ouvrent une brèche à une intervention toujours bien ouverte et renouvelée de l'interprétation de l'œuvre par le lecteur.

Ces nouvelles, quoique pas très connues du grand public, réservent un rôle bien particulier du lecteur dans la recomposition du sens de l'histoire: Celui- ci reste toujours ouvert et susceptible à recevoir bien d'interprétations, toujours plausibles.

Mots clés : Roman policier, Lecteur, Critique , énigme , détective , psychanalyse.

* Professeur adjoint, Département de française, Faculté des Lettres, Université de Tanta

(Les enjeux critiques du roman policier...) Dr. Ahmed Fathy Rezk 


\section{Introduction :}

Dans quelle mesure le roman policier offre-t- il un exemple de la relation lecture/ critique, très à la mode ces derniers temps dans le domaine des études littéraires, suite à l'annonce de la mort de l'auteur par R. Barthes, et le rasement provoqué par le structuralisme de tout discours littéraire centré autour du Sujet, l'auteur; Et sur quels axes s'articule cette relation?

Ces questions constituent la problématique de cette présente recherche qui ne se veut absolument pas une étude exhaustive de la question, vu la vocation et le nombre de pages limités de cette recherche, mais plutôt un approfondissement de certains de ses cotés.

Si le roman policier n'a pas la dignité des autres formes du roman, à cause de l'aspect mécanique et un peu répétitif de son écriture, il n'en reste pas moins un genre littéraire très attirant pour les lecteurs représentatifs de tout âge et de différentes couches sociales. C'est probablement parce qu'un roman policier est facile à lire, mais surtout parce qu'il comporte une énigme qui tient le lecteur à bout de souffle dès le début à la fin du roman.

L'énigme constitue le socle du roman policier, le mystère, une sorte de labyrinthe, que le lecteur, en acceptant le jeu dès le début, essaye de jouer.

En plus la fin du roman policier oriente, le plus souvent, le développement des événements et justifie ses débuts. C'est-à -dire que notre lecture du roman policier, qui comporte le

(Les enjeux critiques du roman policier...) Dr. Ahmed Fathy Rezk 
déchiffrement de son sens et l'interprétation de son énigme, est prédéfinie par l'auteur lui-même, qui oriente, sans qu'on ne se rend pas compte, l'action et par conséquent, le développement de l'histoire. Nous sommes donc, en tant que lecteurs, dupes de notre liberté et de notre rôle à reconstituer l'histoire et à résoudre l'énigme; La questio

majeure qui s'impose à l'esprit maintenant est de savoir si l'auteur, à la lumière de cet exemple du roman policier, a véritablement disparu comme acteur du premier rang du roman? En tout cas, notre participation en tant que lecteurs à reconstituer l'histoire et à résoudre son énigme est un jeu que nous acceptons de jouer comme c'est souligner auparavant.

C'est ce que montre superbement la nouvelle Un drame bien parisien, d'Alphonse Allias (1) .Cette nouvelle suppose en fait, deux lecteurs :le premier naïf et dupe n'arrive pas à déchiffrer le mystère. Mais une deuxième lecture, plus attentive aux petits signes, qui du premier abord n'ont pas de sens, arrive à tout dévoiler.

La petite nouvelle d'Henry James, intitulée, Le motif dans le tapis, ${ }^{(2)}$ et celle d'Edgar Poe, La lettre volée, ${ }^{(3)}$ nous offrent un bon exemple de la relation roman policier et critique littéraire, dans la mesure où le narrateur, chez James, et le préfet de police chargé de résoudre le crime chez Poe, soient intelligents, mais incapables de résoudre l'énigme d'où la possibilité toujours ouverte au lecteur de s'intervenir et de proposer des solutions. 
C'est la raison pour laquelle nous avons choisi de jeter un peu de lumière, quoi que très brève, sur ces deux nouvelles.

En fin de compte, et à travers l'exemple du roman policier, là où on voit certes, le rôle majeur du lecteur dans l'interprétation de l'œuvre, nous posons la question, quand même, de savoir si l'auteur qui définit, sans qu'on s'en aperçoive, tout le processus d'interprétation, et qui joue la fonction de Dieu, présent et absent à la fois, est véritablement mort ?

\section{1-Un détective nommé "lecteur":}

L'importance du roman policier, et qui provient, probablement, de ce que Poe appelait 'les nouvelles de ratiocination" $^{\prime(4)}$ ne doit pas être considérée comme simple phénomène anecdotique.

Le roman policier, même si il n'est pas considéré comme un genre littéraire à part entière, mais à la périphérie de la littérature, garde cependant ses propres caractéristiques, sa saveur à lui, qui est justement l'attente, le fait de tenir le lecteur à l'haleine jusqu'à la fin du roman.

Fondé sur l'attente et l'enquête, il répond en réalité à des pôles d'intérêt très profondément ancrés chez les lecteurs et révèle, avec plus d'acuité que beaucoup d'autres types de textes littéraires, l'importance de la place du lecteur dans la réception et la constitution de 1'œuvre.

En attente, le lecteur est confronté à la combinaison d'éléments qu'il se doit de conjecturer, et qui sont en définitive 
toujours surprenants. Suivant l'enquête, généralement à travers un personnage, il est soumis à l'alternance des lenteurs du cheminement, des impasses du raisonnement et des raccourcis parfois fulgurants de la logique déductive, des associations les plus tenues ou les moins attendues.

Pour parler comme Umberto Eco, qui lui-même s'est illustré avec succès dans le roman d'énigme en écrivant Le Nom la rose (6), l'enjeu est bien de la manière dont le texte présuppose et appelle un « Lecteur modèle » :

< Le lecteur modèle de Edipe [de Sophocle] est appelé à accomplir comparativement les mêmes opérations de reconnaissance des relations qu'CEdipe, en tant que personnage, est invité à accomplir et qu'il accomplit avec quelque retard. Dans ce sens, certains textes narratifs, en racontant l'histoire d'un personnage, fournissent en même temps des instructions sémantico-pragmatiques à leur "lecteur modèle" dont ils racontent l'histoire. II est légitime de penser que cela se passe plus ou moins ainsi dans tout texte narratif et peut-être dans beaucoup d'autres textes non narratifs. $>{ }^{(7)}$.

Le roman policier s'adresse, donc, en l'occurrence, à un certain type de lecteur "prédéfini" par l'auteur, une sorte de lecteur potentiel sur lequel s'appuie toute la force créatrice de l'auteur à accomplir et à recomposer l'histoire.

Par ailleurs, le lecteur du roman policier a, par contrat tacite, une claire conscience de deux éléments: il sera incapable de trouver la solution, et il doit tout faire pour la chercher, à la suite 
de l'enquêteur ou des victimes, selon les cas.

II y a en outre dans la littérature policière une dimension ludique affirmée : il s'agit donc de jouer le jeu et de tenter (en vain) de reconstituer un puzzle, de sortir d'un labyrinthe, de résoudre une énigme, sans que sa propre vie soit engagée bien sûr, puisqu'il s'agit d'un jeu, mais non sans émotions, puisqu'il est difficile d'échapper à toute forme de projection $\left({ }^{8}\right)$.

Dimension ludique encore dans la fascination de la Littérature policière pour la révélation du secret, de l'énigme, de la devinette, des "jeux de l'esprit", comme on appelait au XIXe siècle tous les amusements liés à la manipulation des mots et du langage.

\section{2-Roman policier et psychanalyse:}

La montée en puissance des sciences de l'homme, et surtout de la psychanalyse en tant que discours d'interprétation préalable à la connaissance (et à la thérapie), a considérablement validé les procédures de l'enquête et souligné l'attention qu'il convient d'apporter à l'ambiguïté du langage et aux associations qu'il implique.

Dans de telles conditions, il n'est pas étonnant que la littérature policière ait été l'occasion de croisements particulièrement féconds entre littérature et psychanalyse. ${ }^{(9)}$.

Ainsi Jacques Lacan (1901-1981) a construit l'une de ses plus célèbres études à partir de La Lettre volée d'Edgar Poe ${ }^{(10)}$. Inversement, et peut-être de manière plus surprenante, c'est un «littéraire» comme Pierre Bayard qui entend, par exemple à la 
lecture d'Agatha Christie (11),"appliquer" la littérature à la psychanalyse. II s'agit pour lui de montrer que le texte littéraire permet de comprendre, voire d'enrichir, certaines notions de la démarche psychanalytique. Ceci vaut particulièrement pour le caractère indécidable de l'interprétation.

Pourquoi donc se contenter des seules conclusions du détective, qui sont loin d'épuiser la pluralité des hypothèses que les informations et la logique du texte autorisent à formuler ?

Ainsi, une lecture du roman policier laisse apparaitre l'existence des coupables potentiels mais on ne découvre le vrai coupable qu'à la fin du roman et c'est souvent quelqu'un complètement inattendu.

Cela rappelle que, par définition tout texte, résiste à l'interprétation et que le devoir du lecteur est d'observer les dispositifs de cette résistance.

\section{3-L'énigme conduit la danse:}

L'ambiguïté de la logique du langage, qui dit et masque en même temps, fonde, la légitimité de l'interprète et la nécessité de son activité. C'est vrai pour le rêve, l'énigme et leurs dérives, l'oracle, le jeu de mots, le mot d'esprit, et la devinette. Car même face à la devinette", il y a besoin d'une interprétation qui viendra naturellement trop tard.

La devinette rejoint ainsi le fonctionnement de la figuration, assurant que ce qui apparait impossible le deviendra, à condition que les termes de la "prophétie" soient convenablement

(Les enjeux critiques du roman policier...) Dr. Ahmed Fathy Rezk 
interprétés.

Nous soulignons que le roman policier n'est en réalité que l'expansion, plus ou moins développée de la devinette.

Clément Rosset montre que, dans un roman policier, la réalité, est unique et non double:

< L'évènement attendu vient coïncider avec lui -même, d'où précisément la surprise: car on attendait quelque chose de différent, quoique proche, la même chose mais pas précisément de cette manière. (...) L'évènement qu'on attendait s'est passé mais on s'aperçoit alors que ce qu'on attendait n'était pas cet évènement-ci, mais un même évènement sous une forme différente. On croyait attendre le même, mais en réalité on attendait l'autre. $>\left({ }^{12}\right)$.

\section{4-La fin définit les débuts:}

Qui saurait dans les faits proposer une interprétation pertinente et univoque, sinon celui qui a construit l'énigme ? Du point de vue de la construction littéraire, la devinette, comme le roman policier qui en est l'expansion, repose sur un mode d'élaboration rétrospectif dont Edgar Poe avait défini les grandes lignes:

< S'il est une chose évidente, c'est qu'un plan quelconque, digne du nom de plan, doit avoir été soigneusement élaboré en vue du dénouement, avant que la plume attaque le papier. Ce n'est qu'en ayant sans cesse la pensée du dénouement devant les yeux que nous pouvons donner à un plan son indispensable

(Les enjeux critiques du roman policier...) Dr. Ahmed Fathy Rezk 
physionomie de logique et de causalité - en faisant que tous les incidents, et particulièrement le ton général, tendent vers le développement de l'intention. $>>\left({ }^{13}\right)$

Et, constatant "une erreur majeure dans la méthode généralement utilisée pour construire un conte“, Poe dénonce la pratique de la plupart des écrivains qui se contentent de choisir un sujet sans prendre en compte, dès le stade de l'élaboration du plan, la nécessite de définir «un effet à produire»:

$<$ Ayant toujours en vue l'originalité "car il est traitre envers lui-même celui qui risque de se passer d'un moyen d'intérêt aussi évident et aussi facile", je me dis avant tout: Parmi les innombrables effets ou impressions que l'intelligence ou, pour parler plus généralement, l'âme est susceptible de recevoir, quel est 1'unique effet que je dois choisir dans le cas présent? $>$ (14)

Construire le texte en fonction de l'effet à produire, c'est bien inscrire le lecteur dans le projet d'écriture. Au-delà du débat sur l'inconscient de l'écrivain ou sur la notion plus récente et plus productive $\mathrm{d}^{\prime} \ll$ inconscient du texte ${ }^{(15)} »$, le propre de la littérature oraculaire, énigmatique ou policière et, finalement, la caractéristique de la littérature dans son ensemble est bien de rappeler que chaque œuvre littéraire reste un mécanisme tout tendu vers sa réception.

\section{5-Le lecteur et son double:}

Analysant et reproduisant Un drame bien parisien, brève 
fantaisie d'Alphonse Allais (1855-1905) Umberto Eco montre le caractère exemplaire des dispositions de cette œuvre, qui suppose chez chacun d'entre nous au moins deux lectures, et deux lecteurs successifs: Le premier, nécessairement naif, quelles que soient son intelligence et sa culture, est destiné à être égaré, trompé, parce que le texte d'Alphonse

Allais l'induit sans cesse à se perdre lui-même en ajoutant des informations de son cru destinées à assurer la lisibilité du récit.

Le second lecteur (celui de la relecture) trouvera son plaisir dans l'analyse des erreurs interprétatives du premier et dans la mise à jour de la véritable cohérence d'un drame bien parisien, comme dans sa capacité à créer illusions et pièges logiques.

Et il connaitra un redoublement de jouissance ambiguë lorsqu'il sera, à son tour, gentiment puni par Umberto Eco pour "excès de coopération": > En réalité, il [Un drame bien parisien] appartient à un club raffiné, le club des textes qui racontent des histoires sur la manière dont les histoires se font. Et ce sont des textes beaucoup moins inoffensifs qu'il n'y parait: leur objet critique, c'est la machine de la cul ture, cellelà même qui permet la manipulation des croyances, qui produit les idéologies et titille la fausse conscience qui pe rmet de nourrir sans s'en apercevoir des opinions contradictoires.>> (16)

(Les enjeux critiques du roman policier...) Dr. Ahmed Fathy Rezk 


\section{6-Enjeux de lecture et de critique dans la nouvelle "Le motif} dans le tapis d" Henri James:

Ces capacités de développement de l'attitude critique que la littérature provoque chez son lecteur ne se limitent pas aux textes d'humour auxquels fait allusion Umberto Eco. Ainsi un récit comme Le Motif dans le tapis d' Henry James (1843-1916) expose-t-il admirablement les enjeux de la lecture et de la critique et, au-delà, de la littérature elle-même. C'est pourquoi nous l'avons choisi comme un exemple de cette relation lecteur/ critique. Or un petit rappelle de son histoire est nécessaire pour la compréhension des analyses ultérieures. Le narrateur de ce court récit est un critique littéraire anglais doué et bien introduit, qui échoue à saisir le sens de l'œuvre d'un écrivain au sommet de sa gloire, Hugh Vereker. Sollicité par son ami George Corvick, lui aussi critique, pour écrire un article sur le dernier livre de Vereker, il est invité à rencontrer ce grand homme lors d'une réception mondaine.

L'émotion du débutant qui espère avoir «percé à jour » Vereker (telle était la tâche que lui avait assignée Corvick) tourne vite à la déception quand ce dernier affirme, sans savoir que l'auteur de l'article est à la même table, qu'il s'agit là non pas d'un « dévoilement» mais des «balivernes habituelles ».

Apprenant à peu après l'identité du narrateur, Vereker cherche à l'apaiser, le suit dans sa chambre et, le traitant « d'égal à égal», lui rappelle qu'il est "passé à côté" et lui confie pour tâche

(Les enjeux critiques du roman policier...) Dr. Ahmed Fathy Rezk 
de faire partie du nombre des «initiés» en exhibant la cohérence secrète de l'œuvre prise dans son ensemble:

< II y a dans mon ouvre une idée sans laquelle je n'aurais jamais éprouvé le moindre intérêt pour ce travail. C'est le dessein le plus subtil, le plus abouti de tous, et je crois que son exécution a demandé des trésors de patience, d'ingéni osité. Je devrais laisser quelqu'un d'autre le dire; mais le fait que personne ne le dise est précisément ce dont nous parlons. >> (17)

La conversation se prolonge et finalement Vereker conseille au jeune homme d'« abandonner ». Commence alors, une quête conjointe du narrateur, de Corvick et sa fiancée Gwendolen, une enquête (une chasse au «tuyau ») au cours de laquelle le narrateur rencontre une fois encore Vereker qui lui conseille de nouveau d'abandonner:

A n'en pas avoir des doutes, ce qui déconcertait était pour lui d'une évidence claire. C'était, on l'imaginait, quelque chose qui avait à voir avec le plan original ; comme une image complexe dans un tapis persan. II approuva pleinement cette image lorsque on l'utilisait, et il en utilisa lui-même une autre : 'II s'agit du fil même sur lequel mes perles sont enfilées ${ }^{(18)}$.“

Dans cette traque, dans cette course à l'initiation, le narrateur piétine alors que Corvick, parti comme reporter aux Indes, déclare avoir trouvé la solution et pouvoir désormais se marier avec sa

(Les enjeux critiques du roman policier...) Dr. Ahmed Fathy Rezk 
fiancée, elle-même auteur, qui se fait l'interprète du processus de découverte des Indes.

II ne s'y est pas plongé [dans les livres de Vereker], on le sait, c'est la chose elle-même, ignorée totalement pendant six mois, qui a simplement sauté sur lui comme une tigresse bondit hors de la foret. II n'avait pas emporté de livres avec lui, délibérément; en fait il n'en aurait pas eu besoin: il connait chaque page, par cœur.

Elles ont toutes muri en lui ensemble, et un jour, quelque part, alors qu'il n'y pensait pas, elles ont formé, dans toute leur superbe complexité, la bonne combinaison. Le motif dans le tapis est apparu. II savait que cela viendrait de cette façon [...] ${ }^{(19)}$.

Rien, en particulier lorsque vous vous trouviez en sa présence, ne pouvait se montrer plus parfaitement accompli. Une fois qu'il apparaissait, il apparaissait pour de bon, il était présent avec une splendeur qui vous faisait honte; et, hormis l'insondable vulgarité de l'époque, ou chacun manquait de gout, était perverti ou insensible, il n'y avait pas la moindre raison que cela vous eut échappé. C'était grandiose, et pourtant si simple, et pourtant grandiose, et le savoir était une expérience tout à fait à part ${ }^{(20)}$.

Seuls Corvick (à qui Vereker a confirmé la justesse de sa découverte) et Gwendolen, désormais mariés, peuvent le contempler, Mais Corvick meurt en voyage de noces, sans avoir pu rédiger un article ou exposer sa «trouvaille » et «dévoiler l'idole» alors que le narrateur, éloigné de Londres, n'avait pu le 
rencontrer à son retour en Angleterre. Vereker meurt à son tour. Le narrateur essaie en vain d'épouser Gwendolen, espérant pouvoir accéder au secret qu'il n'a pas évidemment pas trouvé dans un livre qu'elle vient d'écrire. Gwendolen épouse un autre critique, dont elle a deux enfants avant de mourir elle-même en couches.

Et lorsque le narrateur rencontre son confrère veuf un an plus tard, c'est pour apprendre, Mais le «trésor enfoui» ne sera pas pour autant révélé au narrateur. Tout d'abord parce qu'il ne peut tenir dans une lettre et qu'il est en définitive intransmissible, immédiat. Ce que déclare Gwendolen en rapportant les termes d'une lettre de Corvick:

$<<$ médiocre consolation, que lui aussi a été impuissant dans la quête du secret que jamais Gwendolen ne lui a révélé et dont elle était restée l'unique dépositaire.>> (21)

Maintes fois commentée, cette nouvelle, au-delà de sa finesse d'écriture dans la révocation des milieux littéraires et dans l'économie de l'analyse psychologique, apparait bien comme une mise en récit des interrogations que posent la lecture et la critique.

On a relevé à bon droit la dimension «policière » de ce récit ou l'on meurt beaucoup et Jacques Leenhardt ne manque pas de mettre en relation l'image dans le tapis et La Lettre volée:

$<$ Dans les deux cas, on a affaire à une enquête et on assiste à l'échec des moyens traditionnels. De même que dans la nouvelle de Poe, l'objet recherché est introuvable parce qu'il est justement

(Les enjeux critiques du roman policier...) Dr. Ahmed Fathy Rezk 
exhibé, de même le «sens» de l'œuvre de Vereker est« grandiose et simple » et n'apparait que grâce à une association d'idées, sans être sollicité de manière directe ou consciente. D'où le fossé qui sépare le narrateur et Corvick, aussi large que celui qui sépare le préfet de police du chevalier Dupin chez Poe. $>\left({ }^{22}\right)$

Le narrateur est un critique parfaitement intelligent, il est évidemment travailleur et cultivé, il apprécie hautement l'œuvre de Vereker, il a une grande finesse psychologique mais ce n'est pas un bon lecteur.

Que lui manque-t-il alors ? Cette capacité d'émotion, cette aptitude à se laisser aller, cette disposition au partage qui caractérisent Corvick et Gwendolen. Ce qui explique que la communauté d'émotion qu'est l'amour (et non la vie conjugale) ait permis à Corvick et Gwendolen de partager un court instant le bonheur de la contemplation.

Faute d'être un bon medium, le narrateur, comme beaucoup de critiques, manque à sa vocation. Or la nouvelle le suggère constamment: le texte ne se suffit pas à lui-même, et a besoin d'interprètes pour se réaliser.

C'est bien pourquoi Vereker oscille entre le désir d'être découvert et le découragement face aux «balivernes habituelles » alors qu'il sait ne pas pouvoir dire son « dessein » qu'il n'a cessé de « hurler a la figure »101 des critiques.

(Les enjeux critiques du roman policier...) Dr. Ahmed Fathy Rezk 


\section{Conclusion:}

Faisons le bilan de notre travail. Le roman policier offre un meilleur exemple de la relation lecture/ critique dans la mesure où il accorde une place prépondérante au lecteur chargé de recomposer l'histoire et de résoudre l'énigme.

Le roman policier représente également le mariage entre roman et critique dans le sens où il accorde une grande dimension à l'intervention des sciences humaines dans l'interprétation de l'œuvre. Nous parlons évidemment de la psychanalyse comme clé de la compréhension du roman policier et du déchiffrement de son énigme.

Loin d'être une relation bien établie, les nouvelles, Un drame bien parisien, Le motif dans le tapis ainsi que La lettre volée, ouvrent une brèche à une intervention toujours bien ouverte et renouvelée de l'interprétation de l'œuvre par le lecteur.

Ces nouvelles, quoique pas très connues du grand public, réservent un rôle bien particulier du lecteur dans la recomposition du sens de l'histoire: Celui- ci reste toujours ouvert et susceptible à recevoir bien d'interprétations, toujours plausibles.

Résumé des trois nouvelles :

Il nous a semblé utile de rappeler, ici, les résumés des trois nouvelles pour que le lecteur puisse mieux suivre nos analyses:

\section{Un drame bien parisien :}

L'écrivain présente un couple, le mari, nommé Raoul et sa femme Marguerite, toujours en conflit conjugal. Ils se rendent

(Les enjeux critiques du roman policier...) Dr. Ahmed Fathy Rezk 
chacun de son côté, sur dénonciation anonyme, à un bal masquée, pour essayer de découvrir l'infidélité de l'autre. Chacun d'eux connaît à l'avance le déguisement de l'autre : Raoul est déguisé en Chevalier et sa compagne, Marguerite en pirogue africaine

Au cours du bal, le Chevalier invite la Pirogue à diner, et elle accepte. Une fois seuls, ils font tomber leur déguisement et "tous les deux poussèrent, en même temps, un cri de stupeur, en ne se reconnaissant ni l'un ni l'autre. Lui, ce n'était pas Raoul. Elle, ce n'était pas Marguerite“. Après cette scène les protagonistes de la nouvelle décident de ne plus se disputer.

L'intérêt de cette nouvelle est qu'elle repose sur un paradoxe de deux contradictions majeures : chaque personnage ne peut être déguisé comme l'attend l'autre, puisque les lettres reçues ne mentionnent que le déguisement du conjoint; ;es personnages ne peuvent être surpris de ne pas être Raoul et Marguerite, puisqu'ils ne les connaissent pas, à quoi s'ajoute l'absurdité volontaire de la conclusion.

\section{La lettre volée :}

Le préfet de police de Paris informe le détective privée Dupin qu'une lettre de la plus haute importance a été volée dans le boudoir royal. Au moment précis du vol, le voleur est connus du policier, mais celui-ci ne peut pas arrêter le voleur.

Malgré des fouilles extrêmement minutieuses effectuées au domicile du voleur, le préfet de police n'a pas pu retrouver la

(Les enjeux critiques du roman policier...) Dr. Ahmed Fathy Rezk 
lettre. Mettre la main sur cette dernière est cependant d'une grande importance, car son possesseur se retrouve en mesure d'exercer des pressions sur le membre de la famille royale à qui la lettre a été volée. Le préfet de police demande de l'aide au célèbre détective.

Quelques semaines plus tard, Dupin restitue la lettre au préfet. Il explique alors au narrateur comment certains principes simples lui ont permis de retrouver la lettre.

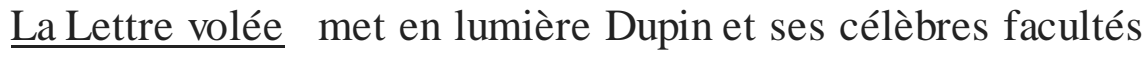
d'analyse. La réflexion logique est au centre de la nouvelle, et toute une part de l'intrigue s'appuie sur les difficultés à trouver une solution rationnelle à la disparition de la lettre.

\section{Le motif dans le tapis :}

Le narrateur de cette nouvelle, un écrivain, se met à chercher un mystérieux motif caché quelque part. Il est rejoint dans sa recherche rejoint par son ami Corvick et la fiancée de ce dernier, Gwendolyn. Un jour, Corvick, en voyage seul en Inde, leur télégraphie qu'il l'a trouvé, mais il meurt avant de leur révéler l'énigme du motif dans le tapis.

(Les enjeux critiques du roman policier...) Dr. Ahmed Fathy Rezk 
1) Alphonse Allais, Euvres anthumes, éd. Robert Laffont, Paris, 1989

2 ) Henry JAMES.; Le Motif dans le tapis, trad, par Elndie Vialieton, Aries. Actes Sud, 1997.

3 ) Edgar. Poe, La Lettre volée. Facultés divinatoires d'August Dupin, traduction par Charles Baudelaire, réd. Gallimard, Paris, 1955.

4 ) Voir Jacques Dubois. Le Roman policier on la modernité, Paris, Nathan, 1996, coll. «Le texte à œuvre ».

5 ) Voir Jean-Yves Mollière, Louis Hachette (1800-1864), Le fondateur d' un empire, Paris, Fayard, 1999.

6 ) Umberto Eco, Le Nom de la rose trad, par Jean-Noël Schifano, Grasset \& Fasquelle, 1980: nouv. éd., Paris, Le livre de Poche, 1982.

7 ) Umberto Eco, op. cit., p. 23

8 ) Voir Boileau- Narcejac, Le Roman policier, Paris, Payot, 1964, coll. «Science de l'homme ».

9 ) Sigmund Freud, Le Délire et les rêves dans la «Gradiva » trad, par Paule Arheix, Rose-Marie Zeitlin et Jean Bellemin-Noel, Préface de J.B. Pontalis, Paris, Gallimard, 1951.

10 ) Jacques Lacan, «Séminaire sur La Lettre volée », in Ecrits, Paris, Seuil, 1966; nouv. éd., 1978, Ecrits I, coll. « Points », p. 19-75.

11 ) Pierre Bayard, Qui a vue Roger Ackroyd ?, éd. Minuit, 1998. Je suivais particulièrement ses feuilletons, nommées "Anthologie du mystère" qui me passionnaient particulièrement et qui étaient diffusés par radio du Caire.

12 ) Voir Clément Rosset. Le Réel et son double, Essai sur l'illusion, Gallimard, 1976, p. 39-40.

(Les enjeux critiques du roman policier...) Dr. Ahmed Fathy Rezk 
13 ) Edgar, POE: La Lettre volée. Facultés divinatoires d'August Dupin, traduction par Charles Baudelaire, édition établie et annotée par Y.-G. Le Dante, Gallimard, 1951.

14 ) Poe, (Euvres en prose, trad., par Charles Baudelaire, édition établie et annotée par Y.-G. Le Dante, Gallimard, 1951, coll. «La Pléiade », p. 979-997. p. 984-985.

15 ) Poe a justement été l'objet d'une des premières "applications" de la psychanalyse à la littérature dans les années trente par Marie Bonaparte. Aujourd'hui il est contesté pour sa naïveté, le monumental ouvrage de Marie Bonaparte (Edgar Poe, Etudes psychanalytiques. Denoël et Steele, 1933,2 vol.) demeure un moment important de 1'évolution de la critique littéraire.

16 ) Umberto Eco, Lector infibula, op. cit., p. 284-285.

17 ) Henry James, Le Motif dans le tapis, op. cit., p. 22-23.

18 ) Henry James, Le Motif dans le tapis, ibid., p. 34-35.

19 ) $\underline{\text { Loc. }}-\mathrm{ci}$

${ }^{20}$ ) Henry James, Le Motif dans le tapis, op. cit. p. 53.

21 ) Henry James, Loc. -cit

22 ) Jacques Leenhardt, Henry James, p.152

(Les enjeux critiques du roman policier...) Dr. Ahmed Fathy Rezk 


\section{Bibliographie sommaire}

1- AYME André-Marc Archéologie de la littérature policière, éd. Harmattan Collection Sang Maudit, 2013.

2- BAYARD, Pierre Qui a tué Roger Ackroyd?, éd. Minuit, 1998.

3- BOILEAU Narcejac, Le Roman policier, éd. Payot, coll. "Science de l'homme".1964.

4- Boltanski Luc Énigmes et Complots. Une enquête à propos d'enquêtes, éd. Gallimard, 2012.

5- De LAVERGNE Elsa La Naissance du roman policier français. Du Second Empire à la Première Guerre mondiale, éd. Classiques, Garnier 2009.

6- DUBOIS Jacques. Le Roman policier on la modernité, Paris, éd.Nathan, coll. « Le texte à oeuvre ».1996.

7- ECO Umberto, Le Nom de la rose trad., par Jean- Noel Schifano, Grasset \& Fasquelle, 1980: nouv. éd.. Paris, Le livre de Poche, 1982.

8- FONDANECH Daniel, Le Roman policier, éditions Ellips 2000. - Le Roman policier, Paralittérature, 2005.

9- FOUREZ, Cathy, Martinez Victor Villatte, Raphaël dir. Quand le délit est dans le texte. Le roman policier, une littérature de l'excès? éd. Peter Lang, Amsterdam 2011.

10-FREUD Sigmund, Le Délire et les rêves dans la « Gradiva » de Jenes , trad., par Paule Arheix,Rose-Marie Zeitlin et Jean Bellemin-Noel, Préface de J.-B. Pontalis, éd. Gallimard, 1985. - L'Interprétation des rêves, traduction par Ignace Meyerson. Révisée par Daniel.Berger. Paris, PUF, 1987. - .Le Mot d'esprit et sa relation à I 'inconscient, traduction

11-MESSIER Denis, préface par Jean-Claude Lavie, éd. Gallimard, coll. « Folio Essais ».Paris, 1998. 
12-JAMES Henry. Le Motif dans le tapis, trad, par Elndie Vialieton, éd. Actes Sud, 1997.

13-LACAN Jacques, «Séminaire sur La Lettre volée », in Ecrits, éd. Seuil, Paris, 1966.

14-MARICOURT Thierry_ Guide du roman policier nordique, éd. Encrage, 2010.

15-MESPLEDE Claude, Dictionnaire des littératures policières, nouvelle édition, Nantes, 2007.

16-MESSAC Régis, $\underline{\text { Roman policier, fragment }}$ d'histoire, éditions Gallimard, Paris, 2009.

17-Perrin Raymond, Histoire du polar jeunesse, romans et bandes dessinées, éd. Harmattan, Paris, 2011.

18-NICOMEDE Béatrice, Le roman policier pour la jeunesse, bonne ou mauvais lecture ?,éd, Harmattan, Paris, , 2012.

19-POE Edgar, La_Lettre volée. facultés divinatoires d'August Dupin, traduction par Charles Baudelaire, Euvres en prose, Gallimard, 1951.

20-REGARD, Frédéric, Le Détective était une femme. Le polar en son genre, éd. PUF, Paris, 2018.

21-ROSSET Clément. Le Réel et son double, Essai sur l'illusion, Gallimard, Paris,1976,

22-TULARD Jean, Dictionnaire du roman policier, éd., Fayard, Paris 2005.

23-VANONCINI André, Le Roman policier, P.U.F, coll. Que sais-je2003

\section{$\underline{\text { Articles : }}$}

- DUBEUX Albert, Le Roman policier, in La revue des deux mondes, $\mathrm{n}^{\mathrm{O}} 16,15$ août 1959.

- Le Roman noir américain, revue Europe, août-Septembre 1984.

(Les enjeux critiques du roman policier...) Dr. Ahmed Fathy Rezk 
- VANONCINI André, «Du roman policier au roman de l'homme: La Nuit du carrefour de Georges Simenon, , Colloque de l'Association internationale des études françaises, 22 juillet 1987 ,

- Cahiers de l'A.I.E.F., mai 1988, n 40; «Narratologie et herméneutique: le roman policier de Simenon au carrefour de la critique »

- Cahiers d'histoire des littératures romanes, 1988, 1/2; "Léo Malet et Charles Baudelaire: de l'enquête policière à la quête poétique », Poétique, 110, avril 1997

- «Roman policier», "Léo Malet», Dictionnaire des lettres françaises, $\mathrm{XX}^{\mathrm{e}}$ siècle, "Le livre de poche", 1998.

- "Le roman policier comme enjeu littéraire», ARBA 10, juin 1998.

- «De Zadig à Maître Cornélius: le roman policier en gestation », Colloque «Lire Balzac en l'an $2000 »$, Paris Saché 18-22 mai 1999, L'Année balzacienne, 1999.

- RAMON Fernandez, Le roman policier in Messages, Paris, 1981. 


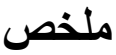

ان الرواية البوليسية هى خير مثنال للالالـة على العلاقـة المعقدة فى الادب بين

القرائة والنقد حيث انها تقتح الباب على مصراعية للقارىه لكي يفسر احداث الروايـة ويحل لغزهـا وبذلك يصبح هذا القارىء محورا للعمل الادبـى. وتعد الروايـة البوليسية

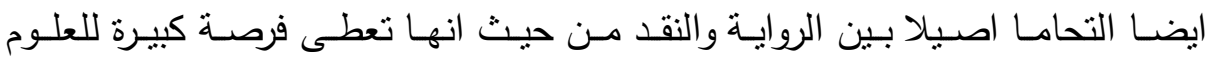
الانسانية من علم النفس وعلم الاجتماع وعلوم اللغة والعلوم السيميائية الخ لكي تقسر العمل الادبى وتحلله فى إطار دور نقدي رائد. ونحن نخص بالذكر علم النفس الذي يلعب دورا كبيرا فى ذلك.

ان تحليلاتتـا لثلاث قصص قصيرة والتى القت الضوء قبل كل شىء على دور القارىء فى تقسير العمل الادبى واعطائه معانيه وتقسيراته المختلفة هو محور هذا البحث. هذه القصص القصيرة هى ماسـاة باريسية للكاتب الفونس اليا وتطريز السجاد لهنرى جيمس والخطاب المسروق لادجار بو. ان هذه القصص القصيرة تلقى الضوء على القارى الذي يقوم بـدوره باعطساء المعنى للاحداث حيث انـه مـن خـلال القراءة يكون أكثر من معنى ويعطى أكثر من احتمال فى عمل دائما منفتح على احتمالات لا تتنهي. الكلمات الدالة: رواية بوليسية، قارى،، ناقد، لغز، محقق، علم النفس 